\title{
Molecular Study of Efflux Genes in Pseudomonas aeruginosa Isolated from Clinical Samples
}

\author{
Maysaa El Sayed Zaki ${ }^{1}$, Ahmed Elewa ${ }^{1}$ and Nashwa M. Al-Kasaby ${ }^{2 *}$ \\ ${ }^{1}$ Clinical Pathology department Faculty of Medicine, Mansoura University, Egypt \\ ${ }^{2}$ Medical Microbiology and Immunology Department, Faculty of Medicine, Mansoura \\ University, Egypt \\ *Corresponding author
}

\begin{abstract}
A B S T R A C T
Pseudomonas aeruginosa is a common nosocomial pathogen associated with several clinical infections. It shows high resistance to most antibiotics classes due to multiple antibiotic resistance mechanisms. The aim of the present study was to assess the presence of efflux system genes by multiplex PCR among clinical isolates of $P$. aeruginosa. The

\section{Keywords}

Multiplex PCR, Pseudomonas aeruginosa, Efflux genes.

Article Info

Accepted:

31 June 2017

Available Online:

10 July 2017 study was conducted on clinical isolates of $P$. aeruginosa collected from Mansoura University hospital from January 2016 to December 2016. Identification of $P$. aeruginosa was performed according to standard microbiological techniques and antibiotics susceptibility was determined by disc diffusion methods. Multiplex PCR was used for amplification of MexA, MexB, and MexR genes. For detection of OprM and $O p r D$ genes, two separate rounds of PCR were performed. Isolated $P$. aeruginosa showed high resistance to gentamicin (90.6\%), amikacin (66.03\%) ceftazidime (77.4\%), Aztreonam $(64.2 \%)$, imipenem $(47.2 \%)$ and ciprofloxacin $(26.4 \%) . P C R$ study for efflux genes revealed the presence of genes in $66.03 \%$ of $P$. aeruginosa isolates; combined MexB and $O p r D$ in $60.4 \%, M e x B$ alone, and combined $M e x B$ and $O p r D$ in $1.9 \%$ for each. The presence of efflux genes was associated significantly with high resistance to gentamicin, ceftazidime, Cefepime, Aztreonam, Ciprofloxacin, Amikacin, Meropenem and Imipenem ( $P$ value $0.0001,0.003,0.0001,0.008,0.02,0.03,0.0001$ and 0.0001 ; respectively). The present study highlights the importance of genes controlling efflux pumps as an important cause associated with $P$. aeruginosa resistance to antibiotics. Longitudinal large scales studies are required for further analysis of these genes and its expression effects on antibiotics resistance of $P$. aeruginosa.
\end{abstract}

\section{Introduction}

Infection with Pseudomonas aeruginosa $(P$. aeruginosa) is common in hospital acquired infections. It can cause a wide spectrum of infections, particularly in immune compromised patients (Aksamit, 1993).

P. aeruginosa has various mechanisms of resistance to antibiotics, such as broad spectrum B-lactamases and metallo- $\beta-1$ actamases (MBL), through the alteration of penicillin-binding proteins (PBP), porin mutations, plasmid enzymatic modification, DNA-gyrase mutations, and active efflux pumps (Garza-Ramos et al., 2009)

The efflux mechanisms are controlled by Resistance-Nodulation-Cell Division (RND) family operon. These RND operons are 
encoded by the bacterial chromosomes. $P$. aeruginosa genome contains at least 12 structural genes for the RND efflux systems, of which four are clinically important: MexAB-OprM ( $\beta$-lactams), MexCD-oprJ and MexEF-oprN (carbapenems and quinolones) and mexXY-oprM (aminoglycosides) (Poole, 2001).

However, different types of efflux pumps may extrude not only antibiotics within the same class but also different classes of antibiotics (Lister, et al., 2009).

These genes are expressed at relatively low levels, under the control of regulatory genes. Mutations in these regulators can lead to raised expression and antibiotic resistance (Sevillano et al., 2006).

Location of efflux resistance is necessary for the selection of antibiotics and its doses for treatment of infections caused by $P$. aeruginosa and for monitoring of antibiotics resistance mechanisms (Morita et al., 2012).

There are both phenotypic methods and genotypic methods for detection of efflux system. However, phenotypic methods are inaccurate with variable results (Mesaros et al., 2007). Molecular method basically depends upon the use of Real-Time PCR for gene expression. Although, this method require special equipped laboratory with the use of many probes and limited to study of two Mex systems (MexAB-OprM and MexXY) (Yoneda et al., 2005).Detection of multiple Mex genes utilizing multiplex PCR can be used as a screening tool for detection of efflux system in clinical microbiology laboratories (Mesaros et al., 2007).

The present study aims to study the presence of efflux system genes by multiplex PCR among clinical isolates of $P$. aeruginosa.

\section{Materials and Methods}

This study was conducted on clinical isolates of $P$. aeruginosa collected from Mansoura University hospital from January 2016 to December 2016. Clinical isolates were obtained from wound samples, blood culture and sputum samples.

Bacteriological cultures were performed according to the type of samples.

Identifications of Pseudomonas species were done according to colony morphology, characteristic pigment production of $P$. aeruginosa, gram staining, positive oxidase tests, and API 20 NE (Collee et al., 1996).

\section{Antibiotics susceptibility test}

Antibiotic susceptibility testing of $P$. aeruginosa was performed according to the Kirby-Bauer disk diff usion method. The used antibiotics discs were: amikacin $(30 \mu \mathrm{g})$, gentamicin $(10 \mu \mathrm{g})$, tobramycin, piperacillin $(100 \mu \mathrm{g})$, piperacillin-tazobactam $(100 / 10 \mu \mathrm{g})$ ceftazidime $(30 \mu \mathrm{g})$, cefepime $(30 \mu \mathrm{g})$, imipenem $(10 \mu \mathrm{g})$, Meropenem $(10 \mu \mathrm{g})$, Aztreonam $(30 \mu \mathrm{g})$, ciprofloxacin $(5 \mu \mathrm{g})$, and Colistin $(10 \mu \mathrm{g})$. According to the clinical and laboratory standards institute (CLSI) 2013.

Escherichia coli ATCC 25922 and $P$. aeruginosa ATCC27853 strains (American Type Culture Collection) were used as quality controls.

Plates were incubated at $37^{\circ} \mathrm{C}$ for $16-18 \mathrm{hrs}$. The diameters of inhibitory zones were measured and the results were reported based on the recommendation of (CLSI) 2013.

Multidrug resistant (MDR) isolates were selected according to their non-susceptibility to at least one agent in three or more antimicrobial categories. 
Purified colonies of isolated $P$. aeruginosa were subculture don Brain Heart Infusion (BHI) broth containing glycerol and kept frozen at $-70^{\circ} \mathrm{C}$ for molecular study.

\section{Bacterial DNA extraction}

Three to five colonies were used for DNA extraction by QIAamp DNA Mini Kit (Qiagen, USA) according to the manufacturer's instructions.

\section{PCR amplification of efflux genes}

Multiplex PCR was used for amplification of MexA, MexB, MexR. PCR for genes OprM and $\operatorname{OprD}$ were performed as two separate rounds of PCR using rPSL gene was used as housekeeping control gene.

The method used for amplification and the primers sequences described previously by Arabestani et al., 2015. Primer sequences used in this study are listed in table 1 .

The amplified products were subjected to gel electrophoresis by the use of $1.5 \%$ agarose gel for one hour. 100-bp DNA size marker (Qiagen, USA) was used and the agarose gel was visualized by using UV transilluminator.

\section{Statistical analysis}

Descriptive data were presented as frequencies and percentages via SPSS software version 18. Chi-square test was used to determine any significant difference. $P$ value less than 0.05 was considered statistically significant.

\section{Results and Discussion}

The study included $106 \quad P$. aeruginosa isolates. They were isolated from wounds swabs $(66 \%)$, followed by sputum $(18.9 \%)$ and blood culture (15.7\%).
Isolated $P$. aeruginosa showed high resistance to gentamicin $(90.6 \%)$, amikacin $(66.03 \%)$ ceftazidime (77.4\%), aztreonam (64.2\%), imipenem $(47.2 \%)$ and ciprofloxacin $(26.4 \%)$, table 2.

PCR study for efflux genes revealed the presence of genes in $66.03 \%$ of $P$. aeruginosa isolates (Figure 1).

PCR study for efflux genes revealed the presence of genes in $66.03 \%$ of $P$. aeruginosa isolates. $M e x-B, O p r D$ in $60.4 \%, M e x-B$ alone and combined Mex-B, Opr-M in $1.9 \%$ for each (Figure 2).

The presence of efflux genes was associated significantly with high resistance to gentamicin, ceftazidime, Cefepime, aztreonam, ciprofloxacin, amikacin, meropenem and imipenem ( $P$ value 0.0001 , $0.003,0.0001,0.008,0.02,0.03,0.0001$ and 0.0001 ; respectively) (Table 3 ).

Figure 3 shows positive $P$. aeruginosa strains for $M e x-B$ by PCR.

Pseudomonas aeruginosa represents a common nosocomial pathogen causing various types of infections. This pathogen is known for its association with resistance to a wide spectrum of antibiotics (Poole, 2000).

In the present study, Isolated $P$. Aeruginosa showed high resistance to gentamicin $(90.6 \%)$, amikacin $(66.03 \%)$ ceftazidime (77.4\%), aztreonam (64.2\%), imipenem (47.2\%) and ciprofloxacin (26.4\%).

In previous studies, gentamicin resistance rate from $63 \%$ up to $93.7 \%$, amikacin from $25 \%$ up to $93.4 \%$, I mipenem from $14 \%$ up to $50 \%$ was reported (Shahcheraghi et al., 2003,Kianpouret al., 2010,Anil and Shahid,2013,Nikokar et al., 2013,Chaudhar et al., 2013). In this study, ceftazidime and 
cefepime showed resistance rate of $77.4 \%$ and $75.4 \%$; respectively. In previous studies, the resistance pattern of $P$. aeruginosa toward third and fourth classes cephalosporins have been noticed, especially in samples from wounds and burns (Jazani et al., 2010; Satti et al., 2011). Resistance to extended-spectrum cephalosporins usually is due to the chromosomally mediated type 1 (AmpC) beta-lactamase (Berrazeg et al., 2015).

Table.1 Genes and primer sequences used in the study

\begin{tabular}{|l|l|l|}
\hline Gene & Sequence & $\mathrm{bp}$ \\
\hline$M e x-A$ & $\begin{array}{l}\text { F: CTCGACCCGATCTACGTC } \\
\text { R: GTCTTCACCTCGACACCC }\end{array}$ & 503 \\
\hline$M e x-B$ & $\begin{array}{l}\text { F: TGTCGAAGTTTTCATTGAG } \\
\text { R: AAGGTCAC GGTGATGGT }\end{array}$ & 280 \\
\hline$M e x-R$ & $\begin{array}{l}\text { F: GAACTACCCCGTGAATC } \\
\text { R: CACTGGTCGAGGAGATGC }\end{array}$ & 411 \\
\hline$O p r M$ & $\begin{array}{l}\text { F: GATCCCCGACTACCAGCGCCCCG } \\
\text { R: ATGCGGTACTGCGCCCGGAAGGC }\end{array}$ & 247 \\
\hline$O p r D$ & $\begin{array}{l}\text { F: ATCTACCGCACAAACGATGAG } \\
\text { R: GCCGAAGCCGATATAATCAAACG }\end{array}$ & 156 \\
\hline$r P S L$ & $\begin{array}{l}\text { F: GCAAGCGCATGGTCGACAAGA } \\
\text { R: CGCTGTGCTCTTGCAGGTTGTGA }\end{array}$ & 201 \\
\hline
\end{tabular}

Table.2 Antibiotics resistance among $P$. aeruginosa isolates

\begin{tabular}{|l|c|c|}
\hline \multirow{2}{*}{\multicolumn{1}{|c|}{ Antibiotics }} & \multicolumn{2}{c|}{ Resistance } \\
\cline { 2 - 3 } & No & \% \\
\hline Amikacin & 70 & 66.03 \\
\hline Gentamicin & 96 & 90.6 \\
\hline Tobramycin & 90 & 84.9 \\
\hline Piperacillin & 98 & 92.5 \\
\hline Piperacillin-tazobactam & 88 & 83.0 \\
\hline Ceftazidime & 82 & 77.4 \\
\hline Cefepime & 80 & 75.5 \\
\hline Imipenem & 50 & 47.2 \\
\hline Meropenem & 55 & 51.9 \\
\hline Aztreonam & 68 & 64.2 \\
\hline Ciprofloxacin & 28 & 26.4 \\
\hline Colistin & 3 & 2.8 \\
\hline
\end{tabular}

Table.3 Efflux genes association with $P$. aeruginosa isolates resistant to antibiotics $(\mathrm{n}=70)$

\begin{tabular}{|l|l|l|}
\hline Antibiotics & P. aeruginosa isolates with efflux genes & P \\
\hline Amikacin & $26(37.1 \%)$ & 0.03 \\
\hline Gentamicin & $70(100 \%)$ & 0.0001 \\
\hline Tobramycin & $60(85.7)$ & 0.002 \\
\hline Piperacillin & $40(57.1 \%)$ & 0.15 \\
\hline Piperacillin-tazobactam & $18(25.7 \%)$ & 0.6 \\
\hline ceftazidime & $58(82.9 \%)$ & 0.003 \\
\hline cefepime & $56(80 \%)$ & 0.0001 \\
\hline Imipenem & $48(68.6 \%)$ & 0.0001 \\
\hline Meropenem & $48(68.6 \%)$ & 0.0001 \\
\hline Aztreonam & $68(97.1 \%)$ & 0.008 \\
\hline Ciprofloxacin & $18(25.7 \%)$ & 0.02 \\
\hline
\end{tabular}

$P$ value $<0.05$ is considered as statistically significant 
Fig.1 Efflux genes detected in isolated $P$. aeruginosa

II efflux genes no efflux genes

\section{$66.03 \%$}

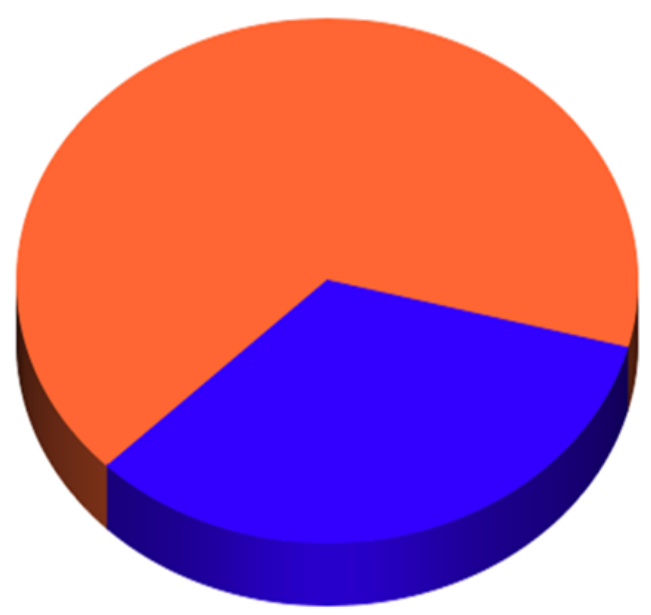

$33.07 \%$

Fig.2 Efflux genes detected by PCR Combined Mex-B, OprD in 60.4\%, Mex-B alone and

Combined $M e x-B, O p r-M$ in $1.9 \%$ for each

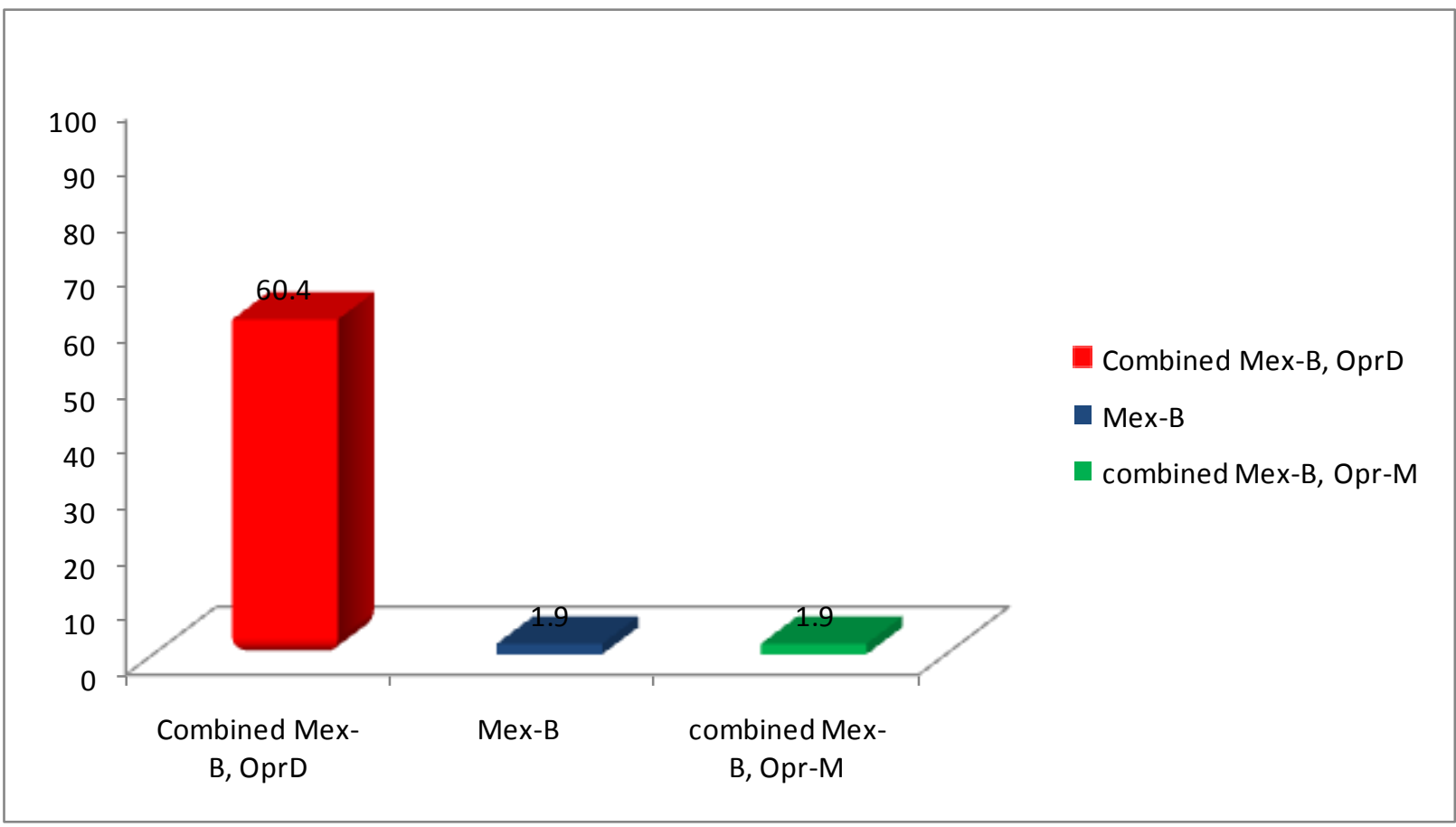


Fig.3 Positive $P$. aeruginosa strains for $M e x-B$

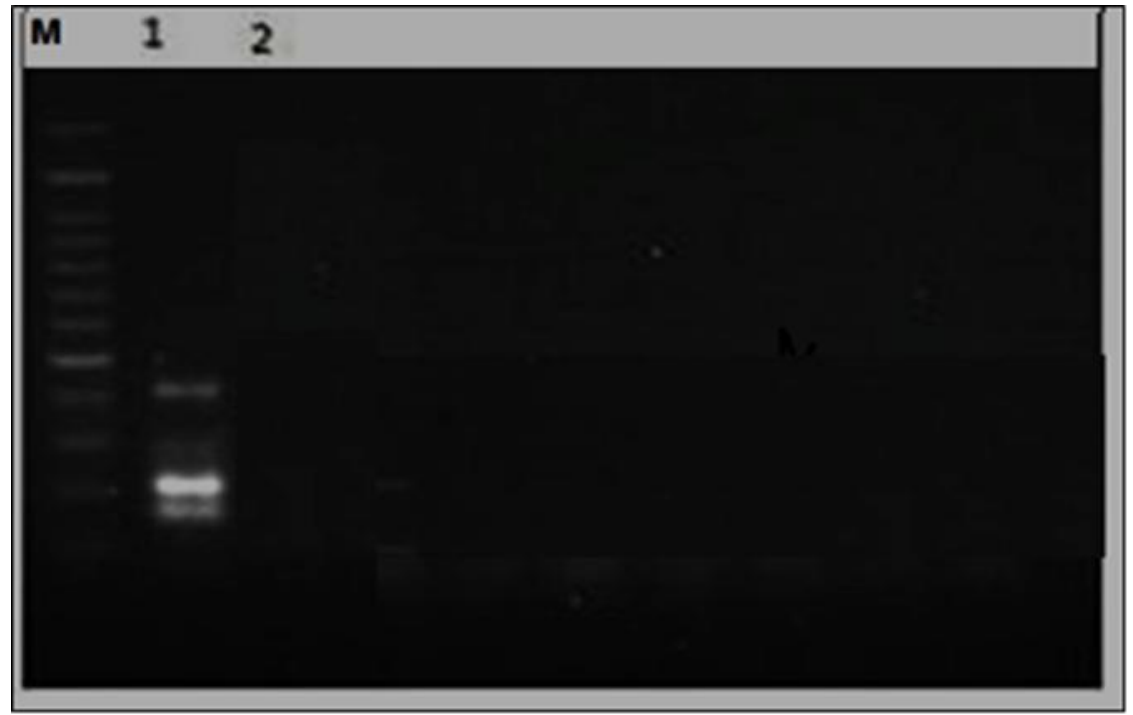

M: Marker; Lane1: positive isolate for Mex- $B$

There are several explanations for high resistance among $P$. aeruginosa strains isolated in the present study. Among the explanations, inappropriate uses of antibiotics in patients either in community acquired infections or in hospital acquired infections leading to selection of antibiotics resistant strains. Meanwhile, $P$. aeruginosa is known to produce over 100 beta-lactamases enzymes leading to marked resistance to beta-lactams antibiotics (Queenan et al., 2010).

The other mechanism associated with antibiotics resistance in $P$. aeruginosa is increased expression of efflux systems MexAB-OprM and MexXY-OprA., repression or inactivation of porin $O p r D$ and this limit the antibiotics entrance to the bacterium cells (Omovskaya et al., 2001)

PCR study for efflux genes revealed the presence of genes in $66.03 \%$ of $P$. aeruginosa isolates; combined MexB, $O p r D$ in $60.4 \%$, $M e x B$ alone and combined MexB and $O p r M$ in $1.9 \%$ for each. The presence of efflux genes was associated significantly with high resistance to gentamicin, ceftazidime, cefepime, aztreonam, ciprofloxacin, amikacin, meropenem and imipenem.
The association of efflux genes and resistance to various antibiotics like gentamicin, Imipenem and meropenem has been described in previous studies (Wang et al., 2010, Morita et al., 2012).

The efflux pumps play a significant role in multiple antibiotics resistance among $P$. aeruginosa species. These structures act by increasing the MIC concentrations of bacterial species, reducing intracellular antibiotics concentrations thus leads to the appearance of resistant strains.

The therapeutic application of efflux pumps inhibitors is a hope for development of new antibacterial therapy among different bacterial species due to the significant structural homology of efflux pumps. Researches have been concentrated on $P$. aeruginosa Mex efflux pumps and their inhibitors. In previous studies, the inhibitor had lowered the MIC values of fluoroquinolones for both sensitive and resistant strains (Omovskaya et al., 2001, Mahmood et al., 2016).

The present study highlights the importance of genes controlling efflux pumps as an 
important cause associated with $P$. aeruginosa resistance to antibiotics. Longitudinal large scale studies are required for further analysis of these genes and its expression effects on antibiotics resistance of $P$. aeruginosa.

\section{Conflicts of Interest}

The authors declare that there are no conflicts of interest regarding the publication of this paper.

\section{References}

Aksamit, T.R., 1992. Pseudomonas pneumonia and bacteremia in the immunocompromised patient. Pseudomonas aeruginosa-The opportunist pathogenesis and disease. CRC, Boston, MA, pp.177-180..

Anil, C. and Shahid, R.M., 2013. Antimicrobial susceptibility patterns of Pseudomonas aeruginosa clinical isolates at a tertiary care hospital in Kathmandu, Nepal. Asian J Pharm Clin Res. 6(3): 235 -238.

Arabestani, M.R., Rajabpour, M., Mashouf, R.Y., Alikhani, M.Y. and Mousavi, S.M., 2015. Expression of Effulux Pump MexAB-OprM and OprD of Pseudomonas aeruginosa Strains Isolated from Clinical Samples using qRT-PCR. Archiv of Iran Med. 18 (2):13-18.

Berrazeg, M., Jeannot, K., Enguéné, V.Y.N., Broutin, I., Loeffert, S., Fournier, D. and Plésiat, P., 2015. Mutations in $\beta$ lactamase AmpC increase resistance of Pseudomonas aeruginosa isolates to antipseudomonal cephalosporins. Antimicrob Agents Chemother. 59(10):.6248-6255.

Chaudhari, V., Gunjal, S. and Mehta, M., 2013. Antibiotic resistance patterns of Pseudomonas aeruginosa in a tertiary care hospital in Central India. Int $\mathbf{J}$ Med Sci Public Health. 2(2): 386-389.

Clinical and Laboratory Standards Institute (CLSI) (2013). Performance Standards for Antimicrobial Susceptibility Testing, Twenty-Third Informational Supplement, M100-S21. Clin Lab Stand Inst 32:1-184.

Collee, J.G., Miles, R.S. and Watt, B., 1996.

Tests for identification of bacteria. Mackie and McCartney practical medical microbiology, 14:13149.

Garza-Ramos, U., Silva-Sánchez, J. and Martínez-Romero, E., 2009. Genetics and genomics for the study of bacterial resistance. SaludPública de México, 51:439-s446.

Jazani, N.H., Babazadeh, H., Sabah, Z. and Zartoshti, M., 2010. The evaluation of antibiotic resistance to cefepime in hospital isolates of Pseudomonas aeruginosa. J. Med. Biomed. 9: 17.

Kianpour, F., Havaei, S.A. and Hosseini, M.M., 2010. Evaluation of Pseudomonas Aeroginosa Isolated from Cutaneous Infections and Determination of Drug Resistance Pattern in Patients of Alzahra Hospital in Esfahan. $\mathbf{J}$ Isfahan Med Sch. 28(110): 1- 6

Lister, P.D., Wolter, D.J. and Hanson, N.D., $2009 . \quad$ Antibacterial-resistant Pseudomonas aeruginosa: clinical impact and complex regulation of chromosomally encoded resistance mechanisms. ClinMicrob Rev. 22: 582610.

Mahmood, H.Y., Jamshidi, S., Mark Sutton, J. and M Rahman, K., 2016. Current advances in developing inhibitors of bacterial multidrug efflux pumps. Curr Med Chem. 23(10):1062-81

Mesaros, N., Glupczynski, Y., Avrain, L., Caceres, N.E., Tulkens, P.M. and Van Bambeke, F., 2007. A combined phenotypic and genotypic method for 
the detection of Mex efflux pumps in Pseudomonas aeruginosa. J AntimicrobChemother. 59(3):378-86.

Morita, Y., Tomida, J. and Kawamura, Y., 2012. MexXY multidrug efflux system of Pseudomonas aeruginosa. Front Microbiol. 28 (3):408.

Nikokar, I., Tishayar, A., Flakiyan, Z., Alijani, K., Rehana-Banisaeed, S., Hossinpour, M., Amir-Alvaei, S. and Araghian, A., 2013. Antibiotic resistance and frequency of class 1 integrons among Pseudomonas aeruginosa, isolated from burn patients in Guilan.Iran J Microbiol. 2013; 5(1): $36-41$.

Omovskaya, O., Warren, M.S., Lee, A., Galazzo, J., Fronko, R., Lee, M., Blais, J., Cho, D., Chamberland, S., Renau, T. and Leger, R., 2001. Identification and characterization of inhibitors of multidrug resistance efflux pumps in Pseudomonas aeruginosa: novel agents for combination therapy. Antimicrob Agents Chemother, 45(1):105-116.

Poole, K. and Srikumar, R., 2001. Multidrug efflux in Pseudomonas aeruginosa components, mechanisms and clinical significance. Curr Top Med Chem. 1(1):59-71.

Poole, K., 2000. Efflux-mediated resistance to fluoroquinolones in gram-negative bacteria. Antimicrob Agents Chemother44: 2233- 2241.

Queenan, A.M., Shang, W., Bush, K. and Flamm, R.K., 2010. Differential selection of single-step AmpC or efflux mutants of Pseudomonas aeruginosa by using cefepime, ceftazidime, or ceftobiprole. Antimicrob Agents Chemother. 54 (10): 4092-4097

Satti, L., Abbasi, S., Qumar, T.A., Khan, M.S. and Hashmi, Z.A., 2011. In Vitro Efficacy of Cefepime against MultiDrug Resistant Pseudomonas aeruginosa-An Alarming Situation in our Setup. The Open Drug Resistance Journal 1: 33-36

Sevillano, E., Valderrey, C., Canduela, M.J., Umaran, A., Calvo, F. and Gallego, L. 2006. Resistance to Antibiotics inClinical Isolates of Pseudomonas aeruginosa. PathologieBiologie (Paris). 54: 493-497

Shahcheraghi, F., Feizabadi, M.M., Yamin, V., Abiri, R. and Abedian, Z., 2003. Serovar determination, drug resistance patterns and plasmid profiles of Pseudomonas aeruginosa isolated from burn patients at two hospitals of Tehran (IRAN). Burns. 29(6): 547 -551.

Wang, J., Zhou, J.Y., Qu, T.T., Shen, P., Wei, Z.Q., Yu, Y.S. and Li, L.J., 2010. Molecular epidemiology and mechanisms of carbapenem resistance in Pseudomonas aeruginosa isolates from Chinese hospitals. J Antimicrobagents. 35(5):486-491.

Yoneda, K., Chikumi, H., Murata, T., Gotoh, N., Yamamoto, H., Fujiwara, H., Nishino, T. and Shimizu, E., 2005. Measurement of Pseudomonas aeruginosa multidrug efflux pumps by quantitative real-time polymerase chain reaction. FEMS microbiology letters. 243(1):125-131

\section{How to cite this article:}

Maysaa El Sayed Zaki, Ahmed Elewa and Nashwa M. Al-Kasaby. 2017. Molecular Study of Efflux Genes in Pseudomonas aeruginosa Isolated from Clinical Samples. Int.J.Curr.Microbiol.App.Sci. 6(7): 4549-4556. doi: https://doi.org/10.20546/ijcmas.2017.607.475 